Cavendish-HEP-97/07

hep-ph/9706263

\title{
Power Corrections in Deep Inelastic Event Shape Variables
}

\author{
Mrinal Dasgupta ${ }^{1}$ \\ Cavendish Laboratory,University of Cambridge,Madingley Road,Cambridge,CB3 0HE,United \\ Kingdom
}

\begin{abstract}
Results are presented for the leading power-suppressed $(1 / Q)$ contributions to shape variables in deep inelastic lepton scattering. These are then combined with available leading order perturbative estimates.
\end{abstract}

\section{INTRODUCTION}

The increasing quantity of data from HERA has given a great boost to the testing of QCD via determinations of the strong coupling constant and the possibility of observing, over a wide kinematic range, its running behaviour.

However in any comparison between theory and experimental data proper account has to be taken of the non-perturbative behaviour inherent in any observable QCD process. While event shape variables are considered useful observables for $\alpha_{s}$ determinations they can suffer from significant power suppressed effects which are non-perturbative in origin. These $1 / Q$ corrections have already been calculated for a number of $e^{+} e^{-}$event shapes [1] and similar results are obtained here for DIS. To make the analogy with $e^{+} e^{-}$shape variables clearer it proves useful to work in the Breit frame and to define current jet event shapes as done in the next section. The dispersive approach [2] is used to estimate the power corrections, which is equivalent to a calculation of the leading renormalon ambiguity.

\section{EVENT SHAPE VARIABLES}

One can define several infrared safe quantities that characterize the shape of the event in the current hemisphere $H_{c}$ of the Breit frame. Perturbative results are available in [3]. The event shapes considered below are the thrust, the jet

1) Talk given at the 5th International Workshop on Deep Inelastic Scattering, Chicago, April 1997. 
mass, the jet broadening and the $C$ parameter with the following definitions in the above order where $\vec{n}$ denotes a unit 3 -vector along the current direction and the summation index $a$ runs over only those partons that are in the current hemisphere.

$$
\begin{gathered}
T_{Q}=\frac{\sum_{a} \vec{p}_{a} \cdot \vec{n}}{Q / 2} \\
\rho_{Q}=\frac{\left(\sum_{a} p_{a}\right)^{2}}{Q^{2}} \\
B_{Q}=\frac{\sum_{a}\left|\vec{p}_{a} \times \vec{n}\right|}{Q / 2} \\
C_{Q}=3\left(\lambda_{1} \lambda_{2}+\lambda_{2} \lambda_{3}+\lambda_{3} \lambda_{1}\right)
\end{gathered}
$$

where the $\lambda_{i}$ denote the eigenvalues of the symmetric matrix $\Theta^{i j}$ defined as

$$
\Theta^{i j}=2 \sum_{a}\left(p_{a}^{i} p_{a}^{j} /\left|p_{a}\right|\right) / Q
$$

The subscript $Q$ indicates the normalization factor $Q / 2$. One can also use a normalization factor given by the sum of energies of partons in the Breit frame, but the normalization does not affect the power corrections we compute.

\section{DISPERSIVE METHOD}

Our estimate of the leading power corrections to the perturbative results are based on the approach of Ref.[2]. Non-perturbative effects at long distances are assumed to give rise to a modification $\delta \alpha_{e f f}\left(\mu^{2}\right)$ in the QCD effective coupling at low values of the scale $\mu^{2}$. The effect on some observable $F$ is then given by a characteristic function $\mathcal{F}(x, \epsilon)$ as follows:

$$
\delta F\left(x, Q^{2}\right)=\int_{0}^{\infty} \frac{d \mu^{2}}{\mu^{2}} \delta \alpha_{e f f}\left(\mu^{2}\right) \dot{\mathcal{F}}\left(x, \epsilon=\mu^{2} / Q^{2}\right)
$$

where

$$
\dot{\mathcal{F}}(x, \epsilon)=-\epsilon \frac{\partial}{\partial \epsilon} \mathcal{F}(x, \epsilon)
$$

The characteristic function is obtained by computing the relevant one-loop graphs with a non-zero gluon mass $\mu[4]$.

Arbitrary finite modifications of the effective coupling at low scales would generally introduce power corrections of the form $1 / k^{2 p}$ into the ultraviolet behaviour of the running coupling itself. As discussed in Ref.[2], such a modification would destroy the basis of the operator product expansion [5]. This leads to the constraint that only terms in the small- $\epsilon$ behaviour of the characteristic function that are nonanalytic at $\epsilon=0$ will lead to power behaved non-perturbative contributions [2]. 

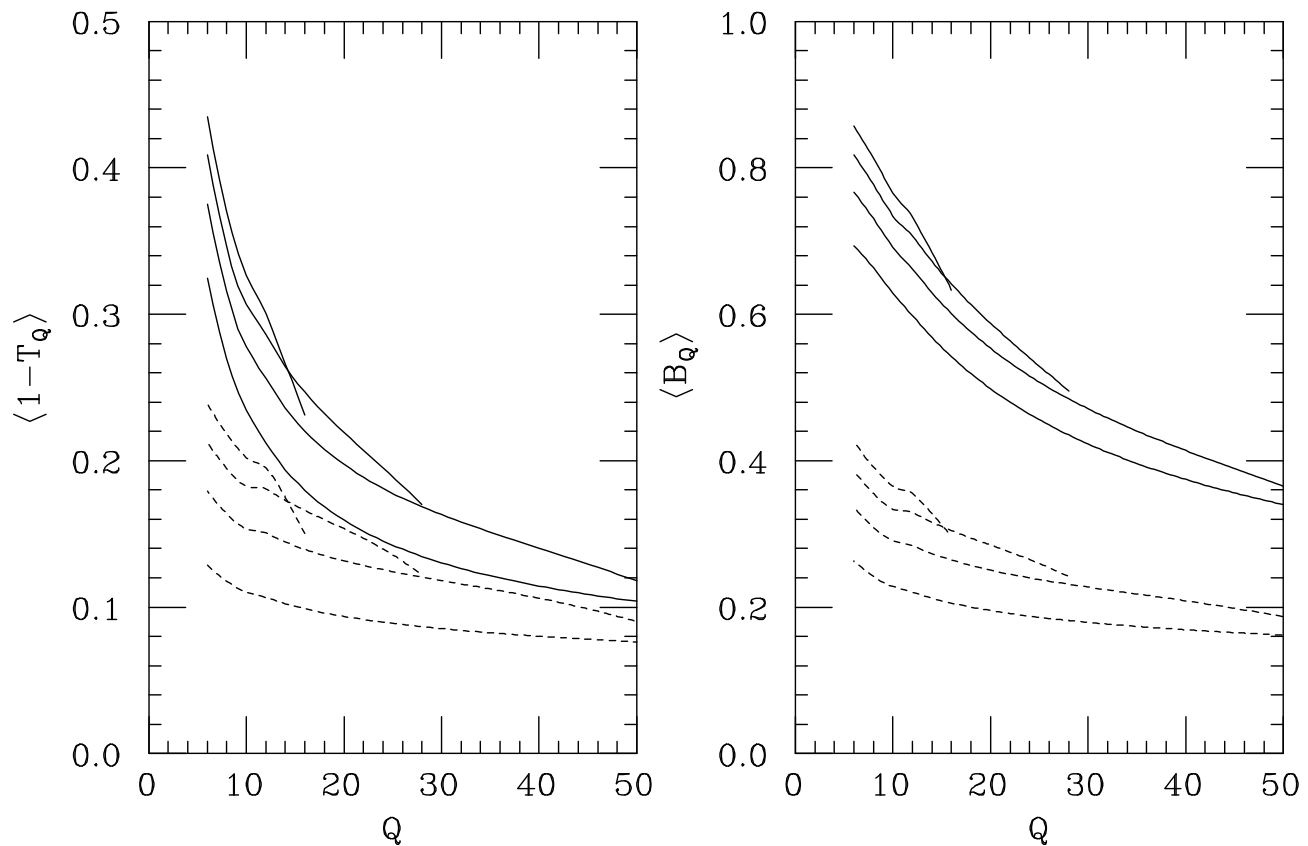

FIGURE 1. Predictions for the mean value of the current jet thrust(left) and the jet broadening in deep inelastic scattering at $\sqrt{S}=296 \mathrm{GeV}$. The 4 plots shown in each case are for $x=0.003$, $0.01,0.03$, and 0.1 . The dashed curves are the leading order perturbative prediction while the solid curves represent the combined leading order perturbative and power behaved contributions

\section{RESULTS}

We find that in DIS as for $e^{+} e^{-}$the leading non-analytic behaviour takes the form of $\sqrt{\epsilon}$ with an extra $\ln \epsilon$ in the jet broadening. The details of the calculation can be found in [3]. The results for the power corrections are presented below. $A_{1}$ is an phenomenological parameter first suggested in Ref.[2] which is expected to be approximately universal. $e^{+} e^{-}$data are consistent with a value for $A_{1}$ of about 0.25 $\mathrm{GeV}$. In the case of the current jet thrust we get a non-perturbative contribution

$$
\delta\left\langle 1-T_{Q}\right\rangle=4 \frac{A_{1}}{Q}
$$

For the current jet mass one gets a correction given by

$$
\delta\left\langle\rho_{Q}\right\rangle=2 \frac{A_{1}}{Q}
$$

For the $C$ parameter we get

$$
\delta\left\langle C_{Q}\right\rangle=12 \pi \frac{A_{1}}{Q}
$$



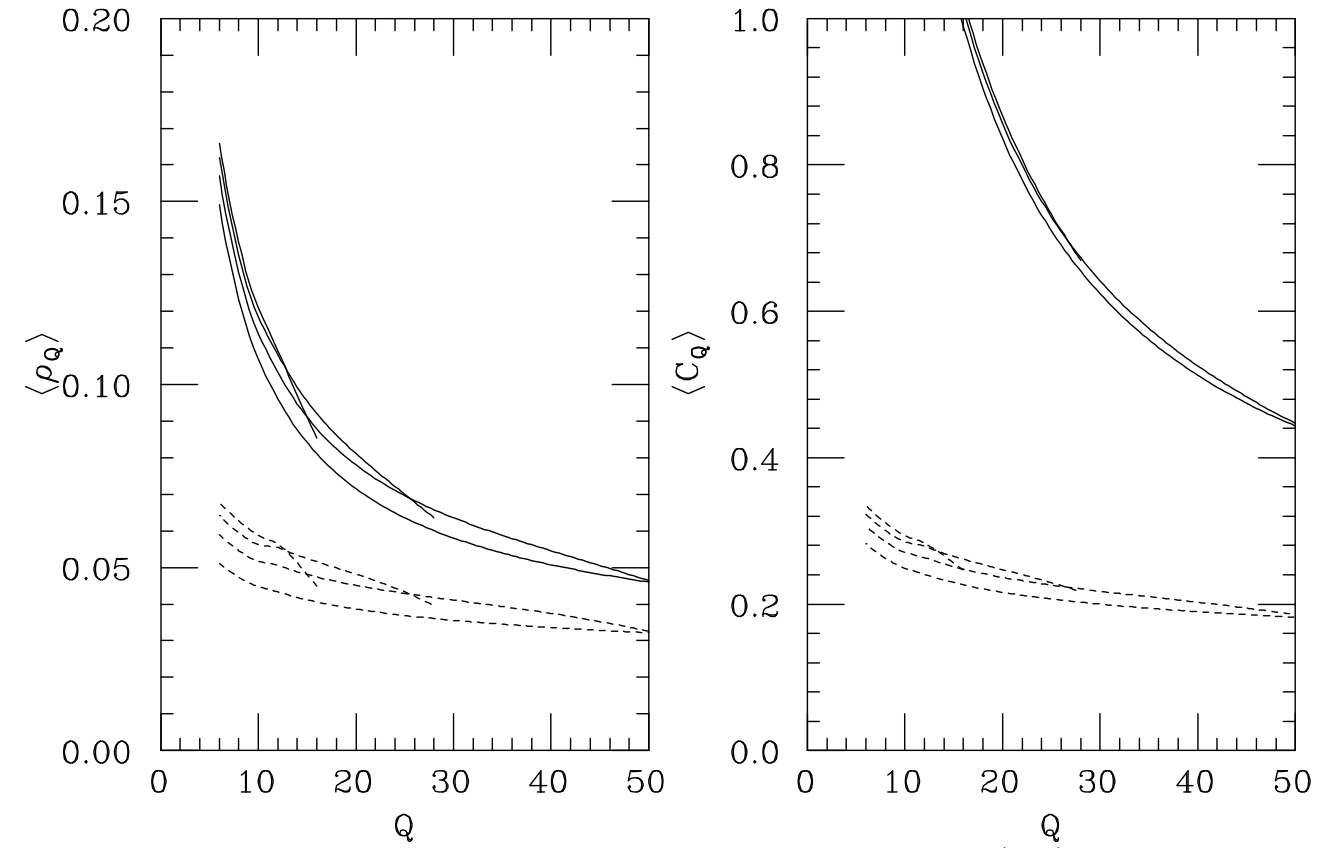

FIGURE 2. Predictions for the mean value of the current jet mass(left) and $C$ parameter in deep inelastic scattering at $\sqrt{S}=296 \mathrm{GeV}$. Curves as in Fig.1

In the case of the jet broadening a slightly different behaviour is obtained

$$
\delta\left\langle B_{Q}\right\rangle=8 \frac{A_{1}}{Q} \ln \frac{Q}{Q_{0}}
$$

where $Q_{0}$ is an undetermined scale. The plots for the combined leading order prediction and power correction are presented for all of these event shape variables in figures 1-2. The MRS $A^{\prime}[8]$ parton distribution was used in every case.

\section{CONCLUSIONS}

It should be pointed out that as explained in Ref.[3] the predictions for the numerical coefficients of $\frac{A_{1}}{Q}$ for the $C$ parameter and the jet broadening are somewhat uncertain. This is due to an inherent limitation of the massive gluon technique with regard to event shapes. The use of a gluon mass in perturbative calculations is equivalent to summing inclusively over all bubbles in the renormalon chain. In the case of less inclusive variables like event shapes the phase space is dependent on the bubble structure which is ignored by the massive gluon formulation. This has been shown for event shapes in [6] and for gluon fragmentation functions in Ref.[7]. It is believed that this limitation does not affect the form of the power corrections to any of the shape variables and in fact appears not to make any significant difference to the predictions for the thrust and the jet mass where our results are not sensitive to the inclusion of a gluon mass in the definition of the shape variable. 
The power corrections here arise from using a massive gluon phase space alone. In the case of the $C$ parameter and the jet broadening the predictions for the numerical coefficients depend on whether or not a gluon mass is included throughout. A glance at the relevant figures also reveals that there are enormously large power corrections in the case of $C_{Q}$ and $B_{Q}$ even at $Q=50 \mathrm{GeV}$. This fact coupled with the uncertainty about the coefficient would mean that these variables are unsuitable for an extraction of the strong coupling. On the other hand the predictions for power corrections to the thrust and the jet mass are more reliable making these, in our opinion, good observables for determination of $\alpha_{s}$. The relevant experimental results have been presented at this workshop by the H1 collaboration $[9,10]$

\section{ACKNOWLEDGEMENTS}

All of the work presented here was done in collaboration with B.R.Webber. I am also grateful to Trinity College, Cambridge and the Committee of Vice-Chancellors and Principals of the Universities of the United Kingdom for financial support.

\section{REFERENCES}

1. B.R. Webber, Plenary talk at the workshop on Deep Inelastic Scattering and QCD, Paris, April 1995.

2. Yu.L.Dokshitzer, G.Marchesini and B.R.Webber, Nucl.Phys. B 469 (1996) 93.

3. M.Dasgupta and B.R. Webber, hep-ph/9704297.

4. B.R Webber, Phys.Lett B 339(1994) 148.

5. M.A Shifman, A.I. Vainshtein and V.I. Zakharov, Phys.Rev.Lett. 73 (1994) 3058.

6. P.Nason and M.H. Seymour, Nucl. Phys. B 454(1995) 291.

7. M.Beneke, V.M. Braun and L.Magnea, hep-ph/9701309.

8. A.D.Martin, R.G Roberts and W.J Stirling, Phys.Rev. D50 (1994)6734.

9. K.Rabbertz, these Proceedings.

10. H1 Collaboration, C.Adloff et al., preprint DESY 97-098, submitted to Phys.Lett.B. 